Ger J Exerc Sport Res 2019 • 49:446-453 https://doi.org/10.1007/s12662-019-00582-5 Received: 13 July 2018

Accepted: 9 April 2019

Published online: 2 May 2019

(c) The Author(s) 2019

O. Roldan-Reoyo ${ }^{1} \cdot$ M. Pelaez $^{2} \cdot$ L. May ${ }^{3} \cdot$ R. Barakat ${ }^{4}$

${ }^{1}$ College of Engineering, School of Sport and Exercise Science, Swansea University, Swansea, UK ${ }^{2}$ College of Sport Science and Physical Activity, European University of the Atlantic, Santander, Spain

${ }^{3}$ School of Dental Medicine, East Carolina University, Greenville, USA

${ }^{4}$ College of Sport Science and Physical Activity, Technical University of Madrid, Madrid, Spain

\title{
Influence of maternal physical exercise on fetal and maternal heart rate responses
}

promote health of the fetus into adulthood as well (Bahls et al., 2014).

Chronic cardiovascular exercise in adults leads to adaptations of the ANS evidenced by lower heart rate $(\mathrm{HR})$ at rest, exercise and recovery (Barbosa, Valente, Sobral, \& Barbosa, 2015; Ostojic et al., 2010). Based on Barker's theory, similar adaptations should occur to the fetus in utero in response to chronic maternal exercise. Research performed in 2010 showed lower HR and increased HR variability at rest, an indicator of improved ANS control, in fetuses from pregnant exercisers compared to fetuses of nonexercisers (May et al., 2010). These findings are similar to the adaptations of an exercise-trained adult (Roldan, Perales, Mateos, \& Barakat, 2015) and suggest an improved functioning of fetal ANS (May, Allen, \& Gustafson, 2016; May et al., 2010).

Other indicators of this beneficial adaptations of the fetal ANS as a consequence of maternal physical activity would further support the Barker theory and the possibility of a fetal training effect from chronic maternal exercise exposure (Roldan et al., 2015). Recovery time after maternal exercise is a feasible variable to measure in the fetus and is known to improve in adults in response to exercise training (Barbosa et al., 2015; Heffernan, Fahs, Shinsako, Jae, \& Fernhall, 2007; Ostojic et al., 2010; Ostojic, Stojanovic, \& Calleja-Gonzalez, 2011). Although fetal heart rate (FHR) recovery time will offer novel insights to adaptations of fetal ANS as a consequence of maternal exercise, this variable has not been evaluated in fetuses as a primary aim.

Thus, the objective of this study is to determine if regular maternal physical activity leads to measurable adaptations of the fetal ANS measured by FHR response recovery time. We hypothesized the FHR recovery would be shorter (quicker) in those exposed to maternal exercise relative to those not exposed to maternal exercise.

\section{Methods}

The present study is part of a randomized controlled trial (ID: RCT, NCT01723293) that was conducted between 2010 and 2015. It was a collaborative effort between the Faculty of Sport Science and Physical Activity of the Technical University of Madrid (Spain) and three local hospitals in Madrid. The study was conducted following the principles of the Declaration of Helsinki.

\section{Study participants}

We recruited pregnant women for this study between September 2011 and January 2015. The study was approved by the Clinical Research Ethics Committee of the University Hospital of Fuenlabrada, Severo Ochoa University Hospital and the University Hospital of Puerta del Hierro. All participants were recruited from obstetrical consulting at these three local hospitals. All women signed written informed consent for inclusion in the 


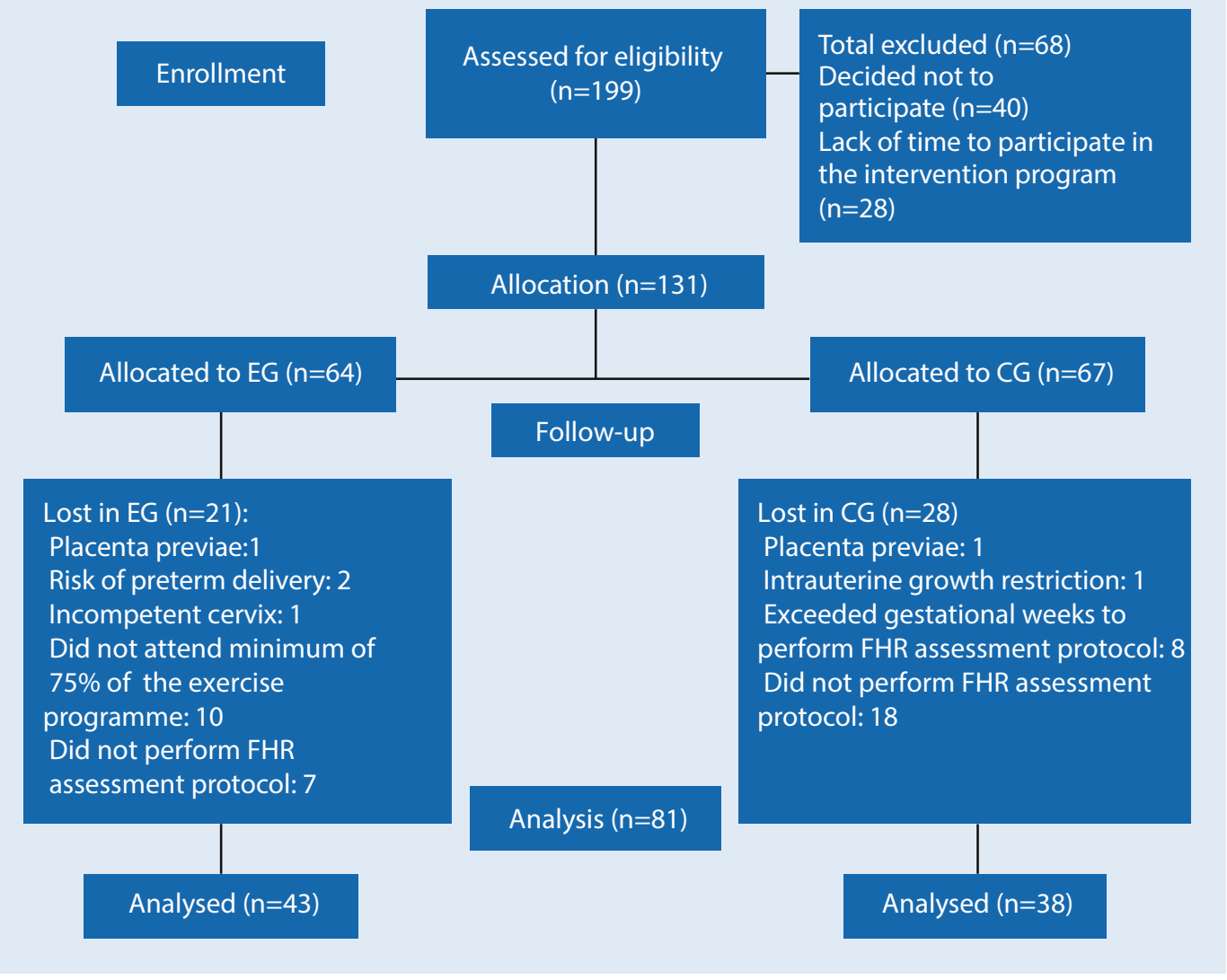

Fig. $1<$ Flow diagram of study participants. FHR fetal heart rate, $C G$ control group, $E G$ exercise group

study; they also completed the participant's information sheet with the study characteristics.

\section{Inclusion criteria}

All participants had no absolute obstetric contraindications to exercise (ACOG, 2015). Women were 25-40 years old and all were living within the community of Madrid. All participants were carrying a single fetus and had no history of alcohol or drug use. Participants were instructed not to participate in other physical activity programs. They were required to give birth at one of the three participating hospitals and were required to understand and speak Spanish fluently. Participants in the exercise group needed to maintain an $75 \%$ adherence to the exercise protocol.

\section{Study groups}

Participants were randomized into two groups: an exercise group (EG) and a control group (CG). EG participants received an intervention based on a physical activity program. CG participants did not receive the intervention and were not encouraged to exercise. All participants had normal obstetric appointments and received the same standard health care.

All participants filled out a demographic questionnaire, which has been used in previous pregnancy studies (Barakat, Pelaez, Lopez, Montejo, \& Coteron, 2012b; Barakat, Cordero, Coteron, Luaces, \& Montejo, 2012a; Pelaez et al., 2011). Within this questionnaire, participants answered questions regarding their physical activity habits before pregnancy (within the year before) and during the first trimester. The same questionnaire was also completed by all participants at 36 weeks of pregnancy. For women in the CG, if the physical activity levels they reported on the questionnaire were above 500 MET min (metabolic equivalent minutes) and/or $150 \mathrm{~min} /$ week of moderate intensity activity, they were withdrawn from analysis. Similarly, only EG partic- ipants that complied $\geq 75 \%$ of attendance were included in the analysis (• Fig. 1).

\section{Physical activity program}

A physical activity program was designed following ACOG guidelines (ACOG, 2002) and the Blooming Exercise Program (Pelaez, 2011), an exercise program developed in Spain specifically for pregnant women. The EG began the program at 10-12 weeks gestation until 36-40 weeks gestation, three days per week. Each session lasted $60 \mathrm{~min}$ and included the following: warm up for 5-7 min, aerobic dance for 25-30 min, strength training for $8-10 \mathrm{~min}$, pelvic floor muscle training for $8-10 \mathrm{~min}$, stretching for 3-5 min.

The intensity was measured using heart rate (HR) target zones from 40 to $60 \%$ reserve heart rate (Zavorsky \& Longo, 2011) for moderate intensity. Participants in EG wore a HR monitor (FT7 Polar Electro Oy, Finland) to ensure training within their target $\mathrm{HR}$ zone. 
Ger J Exerc Sport Res 2019 · 49:446-453 https://doi.org/10.1007/s12662-019-00582-5

(c) The Author(s) 2019

\section{O. Roldan-Reoyo $\cdot$ M. Pelaez $\cdot$ L. May $\cdot$ R. Barakat}

\section{Influence of maternal physical exercise on fetal and maternal heart rate responses}

\section{Abstract}

This study aims to determine if regular maternal physical exercise leads to measurable adaptations of the fetal autonomic nervous system (fANS) tested by fetal heart rate recovery time. A randomized controlled trial enrolled 131 pregnant women into two study groups, Exercise Group (EG) and Control Group (CG). All participants signed an informed consent, study was approved by the local Clinical Research Ethics Committee. Data was analyzed on 81 participants.

EG participants $(n=43)$ received a supervised physical exercise program (PEP) three times a week from 10-12 to 36-40 weeks gestation. CG participants $(n=38)$ did not receive any supervised PEP. All women were tested between 34-36 weeks gestation using two walking tests of three minutes each at $40 \%$ and $60 \%$ maternal heart rate reserve. Resting fetal heart rate (FHR), FHR post-maternal exercise, and fetal recovery time (FRT) in minutes/seconds (m/s) were recorded. Resting FHR was similar between groups $140.88 \pm 9$ EG vs $141.95 \pm 7.35$ CG $(p>.05)$. Difference in resting to post-maternal exercise FHR was statistically significant in fetuses from the CG $(p<.05)$, however was not statistically significant in fetuses from the EG $(p>.05)$. FRT was different between groups after both tests: $40 \%$ test: $2 \mathrm{~m} 15 \mathrm{~s} \pm 3 \mathrm{~m} 19 \mathrm{~s}$ EG vs $7 \mathrm{~m} 6 \mathrm{~s} \pm 5 \mathrm{~m} 28 \mathrm{~s}$
CG ( $p<.001) ; 60 \%$ test: $3 \mathrm{~m} \pm 4 \mathrm{~m} 3 \mathrm{~s}$ EG vs $9 \mathrm{~m} 25 \mathrm{~s} \pm 7 \mathrm{~m} 33 \mathrm{~s} C \mathrm{G}(p<.001)$. There were no significant differences in maternal variables ( $p>.05)$, except for maternal recovery time after $40 \%$ test $(p<.05)$. Influence of maternal exercise in FHR response still remains unclear. Further research is needed to elicit a former conclusion on the effects of maternal exercise on fANS. Overall, exercise during pregnancy is safe and not harmful for the cardiovascular system of the fetus.

Keywords

Physical activity · Pregnancy · Cardiovascular health · Autonomic nervous system · Antenatal training

\section{Einfluss der mütterlichen sportlichen Aktivitäten auf die Reaktion der fetalen und mütterlichen Herzfrequenz}

\section{Zusammenfassung}

Die vorliegende Studie hat das Ziel zu untersuchen, ob regelmäßige sportliche Aktivität von werdenden Müttern messbare Veränderungen im fetalen autonomen Nervensystems (fANS) hervorruft. Dies wurde anhand der fetalen Herzratenerholungszeit getestet. In einer randomisiert kontrollierten Studie wurden 131 schwangere Frauen entweder einer Sportgruppe (SG) oder einer Kontrollgruppe (KG) zugeteilt. Alle Versuchspersonen unterschrieben eine Einverständniserklärung und die Studie wurde durch das lokale Ethikkommission für klinische Forschung genehmigt. Es wurden Daten von 81 Teilnehmerinnen analysiert. Die Teilnehmerinnen der SG $(n=43)$ erhielten jeweils in der 10.-12. und 36.-40. Schwangerschaftswoche drei betreute Sporteinheiten. Die Teilnehmerinnen der Kontrollgruppe $(n=38)$ blieben ohne betreutes Sportprogramm.
Alle Frauen wurden während der 34. und 36. Schwangerschaftswoche getestet. Dazu wurden zwei Geh-Tests à 3 Minuten bei $40 \%$ und $60 \%$ Herzfrequenzreserve durchgeführt. Es wurde die fetale Ruhe-Herzfrequenz (FHF), die FHF nach sportlicher Aktivität der Mutter und die fetale Erholungszeit (FEZ) in Minuten/Sekunden (min/sek) gemessen. Die Gruppen zeigten eine ähnliche Ruhe-FHF: $140,88 \pm 9$ (SG) vs $141,95 \pm 7,35$ (KG) ( $p>.05$ ). Föten in der KG, nicht aber in der SG, zeigten einen signifikanten Unterschied in ihrer Herzfrequenz nach sportlicher Aktivität der Mutter, verglichen mit der Ruhe-Herzfrequenz. Die FEZ der Gruppen unterschied sich in beiden Geh-Tests: $40 \%$-Test: 2 min 15 sek \pm 3 min 19 sek (SG) vs. 7 min $6 \mathrm{sek} \pm 5 \mathrm{~min}$ 28 sek (KG) (p>.001); 60\%-Test: $3 \mathrm{~min} \pm 4$ min 3 sek (SG) vs. 9 min 25 sek \pm 7 min 33 sek (KG) $(p<.001)$. Für die Mütter ergaben sich außer in der Erholungszeit nach dem 40\%-Test $(p<.05)$ in keiner der erhobenen Variablen signifikante Unterschiede ( $p>.05)$. Der Einfluss der sportlichen Aktivität von werdenden Müttern auf die fetale Herzrate bleibt unklar. Weitere Forschung ist erforderlich, um Schlussfolgerungen zu den Auswirkungen der sportlichen Aktivität von werdenden Müttern auf das fANS zu ziehen. Insgesamt betrachtet, ist sportliche Aktivität während der Schwangerschaft als sicher und nicht schädlich für das kardiovaskuläre System des Fötus einzustufen.

\section{Schlüsselwörter}

Körperliche Aktivität · Schwangerschaft . Kardiovaskuläre Gesundheit · Autonomes Nervensystem · Pränatales Training

\section{Fetal heart rate assessment protocol}

All participants performed two walking tests, the first one at $40 \%$ maternal heart rate reserve (HRR), and the second one at $60 \%$ maternal HRR, between 34 and 36 weeks in one of three participating hospitals. These intensity targets were selected because they are the recommended intensity for exercise during pregnancy
(Barakat, Ruiz, Rodríguez-Romo, Montejo-Rodríguez, \& Lucia, 2010; Barakat, Stirling, Zakynthinaki, \& Lucía, 2008; Szymanski \& Satin, 2012; Zavorsky \& Longo, 2011). This target HR zone is safe and is not associated with harmful effects for mother and fetus. Each walking test began with a three minute warm up for safety and to ensure target HR, immediately afterwards the walking test was performed and lasted three minutes.
Test length is short enough for all participants to complete, but long enough to produce maternal and fetal physiological responses (Brenner, Wolfe, Monga, \& McGrath, 1999; Rafla \& Cook, 1999). At 34-36 weeks of gestation, the fetal heart and circulatory system have completed structural changes making heartbeat recordings easier (Sadler, 2010). This period also provides 20 weeks of exercise exposure for the fetus in the exercise 
Table 1 Fetal heart rate (FHR) responses

\begin{tabular}{|c|c|c|c|}
\hline \multirow[b]{2}{*}{ Variable } & \multicolumn{2}{|l|}{ Study group } & \multirow[b]{2}{*}{$p$ value } \\
\hline & CG $(n=38)$ & EG $(n=43)$ & \\
\hline Resting FHR (beats/min) & $141.9 \pm 7.3$ & $140.8 \pm 9.4$ & 0.147 \\
\hline $\begin{array}{l}\text { FHR after maternal exercise } \\
40 \% \text { (beats } / \mathrm{min} \text { ) }\end{array}$ & $147.8 \pm 12.5$ & $139.9 \pm 13.4$ & 0.008 \\
\hline FHR recovery time $40 \%(\mathrm{~min} / \mathrm{s})$ & $7 \min 6 s \pm 5 \min 28 s$ & $2 \min 15 s \pm 3 \min 19 s$ & 0.000 \\
\hline $\begin{array}{l}\text { FHR after maternal exercise } \\
60 \% \text { (beats } / \mathrm{min} \text { ) }\end{array}$ & $148.0 \pm 15$ & $143.7 \pm 10.4$ & 0.134 \\
\hline FHR recovery time $60 \%(\mathrm{~min} / \mathrm{s})$ & $9 \min 25 s \pm 7 \min 33 s$ & $3 \min 0 s \pm 4 \min 3 s$ & 0.000 \\
\hline
\end{tabular}

Table 2 Comparison of fetal heart rate (FHR) at rest and FHR post-exercise within groups

\begin{tabular}{|c|c|c|c|}
\hline CG $(n=38)$ & FHR at rest (beats/min) & $\begin{array}{l}\text { FHR post-exercise } \\
\text { (beats/min) }\end{array}$ & $p$ value \\
\hline Walking test at $40 \%$ & $141.9 \pm 7.3$ & $147.8 \pm 12.5$ & 0.003 \\
\hline Walking test at $60 \%$ & $141.9 \pm 7.3$ & $148.0 \pm 15$ & 0.015 \\
\hline EG $(n=41)$ & FHR at rest (beats/min) & FHR post-exercise (beats/min) & $p$ value \\
\hline Walking test at $40 \%$ & $140.8 \pm 9.4$ & $139.93 \pm 13.48$ & 0.581 \\
\hline Walking test at $60 \%$ & $140.8 \pm 9.4$ & $143.7 \pm 10.4$ & 0.681 \\
\hline
\end{tabular}

Table 3 Maternal heart rate (HR) during fetal HR assessment protocol

\begin{tabular}{|c|c|c|c|}
\hline \multirow[b]{2}{*}{ Variable } & \multicolumn{2}{|l|}{ Study group } & \multirow[b]{2}{*}{$p$ value } \\
\hline & CG $(n=38)$ & $\mathrm{EG}(n=43)$ & \\
\hline $\begin{array}{l}\text { Maternal HR at rest } \\
\text { (beats/min) }\end{array}$ & $88.2 \pm 12.2$ & $87.4 \pm 11.3$ & 0.646 \\
\hline $\begin{array}{l}\text { Maternal HR at } 40 \% \\
\text { (beats/min) }\end{array}$ & $127.9 \pm 7.6$ & $127.2 \pm 7.3$ & 0.596 \\
\hline $\begin{array}{l}\text { Maternal HR recovery at } 40 \% \\
(\mathrm{~min} / \mathrm{s})\end{array}$ & $8 \min 31 s \pm 4 \min 37 s$ & $6 \min 49 s \pm 4 \min 14 s$ & 0.034 \\
\hline $\begin{array}{l}\text { Maternal HR at } 60 \% \\
\text { (beats/min) }\end{array}$ & $147.8 \pm 5.5$ & $147.1 \pm 5.5$ & 0.481 \\
\hline $\begin{array}{l}\text { Maternal HR recovery at } 60 \% \\
(\mathrm{~min} / \mathrm{s})\end{array}$ & $14 \min 3 s \pm 5 \min 32 s$ & $11 \min 49 s \pm 5 \min 16 s$ & 0.058 \\
\hline CG control group, EG exercise g & roup & & \\
\hline
\end{tabular}

group and thus long enough to elicit fetal adaptations (May et al., 2010).

The day of the test women were instructed to eat a snack 90 min before the test. During the first 20 min, resting maternal and FHR were recorded using a HR monitor (FT7 Polar Electro Oy, Finland) and a wireless FHR monitor (Wireless Telemetry T800 Hispania Hospital S.L Spain) respectively (Roldan et al., 2015). Maternal HR at rest was established as the lowest value recorded on the HR monitor during the 20 min resting period. Target HR for each test was obtained using the Karvonen Formula (target heart rate $=(\mathrm{HRR} \times$ intensity of the cellence (RCOG/NICE) guidelines for cardiotocography (CTG) interpretation (Ayres-de-Campos \& Bernardes, 2010; RCOG, 2001).

Once resting maternal HR and baseline FHR were recorded, each participant performed a three-minute warm-up followed immediately by the three-minute walking test at $40 \%$ HRR intensity. During the three minutes walking test, maternal HR was continuously recorded and checked to validate the participant was within the target HR for the given intensity. A range of $\pm 2 \mathrm{bpm}$ was allowed. With the use of wireless sensors, FHR was continuously monitored during this time.

Once the first walking test was finished, FHR was labelled as FHR after maternal exercise. Maternal and fetal HR recovery time were recorded as well. Since it is not recommended to sit immediately after exercise, participants remained standing during the first $5 \mathrm{~min}$ of recovery (ACSM, 2014). After $5 \mathrm{~min}$, participants were able to sit in a comfortable chair. The maximum time for recovery was set at $20 \mathrm{~min}$.

Recovery was defined as the return to resting (baseline) maternal and fetal HR. Maternal HR recovery time was defined as the time required to return to the resting (baseline) HR measured during the initial 20-minute resting period. FHR recovery time was defined as the time elapsed from the end of the walking test to the time that FHR returned to the baseline value. Maternal and fetal recovery time were recorded for $20 \mathrm{~min}$. If FHR did not return to baseline within $20 \mathrm{~min}$ after the $40 \%$ test, then we waited until FHR was at baseline levels or $\pm 5 \mathrm{bpm}$ of baseline before proceeding to the $60 \%$ test. Once this recovery period was completed, the second test at $60 \%$ HRR intensity was performed. The three-minute walking test at $60 \%$ HRR intensity was performed following the same procedure as the first walking test.

Our primary outcome variable was FHR recovery time. The secondary outcome variables were FHR at rest, FHR after exercise, and difference between these timepoints; as well as, percentage of fetuses recovered by $20 \mathrm{~min}$, maternal $\mathrm{HR}$ 
Table 4 Correlation between maternal heart rate (MHR) recovery time and fetal heart rate (FHR) recovery time for both tests

\begin{tabular}{|l|lll|}
\hline & & $\begin{array}{l}\text { Group } \\
\text { CG }(\mathbf{n = 3 8})\end{array}$ & EG $(\boldsymbol{n}=\mathbf{4 3})$ \\
\hline $\begin{array}{l}\text { MHR recovery time } \\
\text { at } 40 \%\end{array}$ & - & $\begin{array}{l}\text { FHR recovery time } \\
\text { at } 40 \%\end{array}$ & $\begin{array}{l}\text { FHR recovery time } \\
\text { at } 40 \%\end{array}$ \\
\hline & Spearman correlation & 0.157 & 0.006 \\
\hline $\begin{array}{l}\text { MHR recovery time } \\
\text { at } 60 \%\end{array}$ & - & $\begin{array}{l}\text { FHR recovery time } \\
\text { at } 60 \%\end{array}$ & $\begin{array}{l}\text { FHR recovery time } \\
\text { at } 60 \%\end{array}$ \\
& & -0.150 & -0.286 \\
\hline CG control group, EG exercise group & & \\
\hline
\end{tabular}

at rest, maternal $\mathrm{HR}$ at after exercise, and maternal HR recovery time.

\section{Statistical analysis}

All statistical analyses were performed using the Statistical program SPSS 20.0 version for $\mathrm{PC}$ with a level $<0.05$ a priori. Shapiro-Wilk test was performed in all variables to test normality of the data. Results from this test showed a non-normal distribution of the data in the following variables: baseline FHR at rest, FHR recovery time in both tests, maternal $\mathrm{HR}$ at rest, maternal $\mathrm{HR}$ at the end of both tests and maternal HR recovery time. Therefore, non-parametric Mann-Whitney U test was performed to analyze differences in variables with nonnormal distributions. Spearman correlations were used to evaluate correlations in maternal and fetal recovery time. T-tests for unpaired samples were performed to analyze mean differences in FHR after exercise between groups. All data are presented as mean \pm standard deviation (SD). Due to signal loos of the FHR during maternal exercise, we were aunable to analyze this variable.

\section{Results}

In all, 199 pregnant women were recruited for the study. All women met inclusion criteria to participate in the study. From the 199 assessed for eligibility, 68 were excluded (• Fig. 1). Of those remaining, 131 were randomized into EG, $n=64$, and CG, $n=67$, (• Fig. 1) following a Simple Random Sampling protocol (Armitage \& Berry, 2002).

From the 131 pregnant women randomized, only 81 were included in data analysis (• Fig. 1). All 81 women had healthy, uncomplicated pregnancies (43 EG, 38 CG). Baseline characteristics (mean $\pm \mathrm{SD}$ ) were similar between groups: age $(32.6 \pm 4$ years in EG; $32.6 \pm 3.5$ years in CG), prepregnancy weight $(60.9 \pm 9.1 \mathrm{~kg}$ in the $\mathrm{EG}$; $62.9 \pm 10 \mathrm{~kg}$ in the CG), and prepregnancy BMI $(22.3 \pm 3.2$ in the EG; $23.4 \pm 3.4$ in CG). All participants delivered healthy babies (41 male vs 40 female): birth weight (g) (3139.00 \pm 476.86 EG vs 3222.41 \pm 416.62 CG; $p>0.05)$; height (cm) $(49.62 \pm 2.61$ EG vs $49.51 \pm 2.41$ CG; $p>0.05)$; head circumference $(\mathrm{cm})$ $(34.34 \pm 1.29$ EG vs $34.60 \pm 1.46 \mathrm{CG}$; $p>0.05)$; Apgar score $1 \mathrm{~min}(8.93 \pm 1.18$ EG vs 9.09 $\pm 0.77 \mathrm{CG}$; $p>0.05)$; Apgar score $5 \mathrm{~min}(9.67 \pm 1.21 \mathrm{EG}$ vs $9.88 \pm 0.42$ CG; $p>0.05)$. Two offspring from EG and one from CG had low birth weight $(<2.5 \mathrm{~kg})$; two offspring from EG were macrosomic ( $>4 \mathrm{~kg})$, none from the CG.

\section{FHR responses}

All baseline FHR at rest values were within the normal healthy range for both groups (110-160 bpm; RCOG, 2001). There were no differences in FHR at rest between groups (• Table 1). FHR recovery time showed significantly quicker recovery times in the exercise group relative to the control group at both intensities (- Table 1).

FHR after three-minute walking test was lower in fetuses from EG, but only significantly lower at $40 \%$ intensity $(p<0.05$; - Table 1). Further analysis was performed to compare resting FHR and FHR immediately after each three-minute walking test within each group. Statistically significant differ- ences were found only for fetuses from CG (• Table 2).

The percentage of fetuses that recovered within 20 min after maternal exercise was analyzed for both three-minute walking tests between groups. Of the fetuses in EG, $100 \%(43 / 43)$ recovered within $20 \mathrm{~min}$ after both walking test. Of the fetuses in CG, $94.7 \%(36 / 38)$ and $81.6 \%(31 / 38)$ recovered within $20 \mathrm{~min}$ after the $40 \%$ intensity and $60 \%$ walking test, respectively. We did not observe any episodes of bradycardia or tachycardia within CTG recordings from those fetuses. However, significantly fewer fetuses from the CG achieved recovery to resting FHR within $20 \mathrm{~min}$ following the $60 \%$ intensity test $(p<0.05)$.

\section{Maternal HR during FHR assessment protocol}

There were no between group differences in maternal $\mathrm{HR}$ at rest, or maternal HR after exercise at $40 \%$ and $60 \%$ intensities (- Table 3). Participants in the EG had significantly quicker maternal HR recovery time after the three-minute walking test at $40 \%$ intensity. Furthermore, there are trends towards significant differences in the maternal HR recovery time after the walking test at $60 \%$ intensity.

Spearman correlation analysis was performed to determine possible relationships between maternal measures and fetal HR recovery time. Results showed no significant correlations in any of the variables or at any intensity ( $p>0.05$; $\bullet$ Table 4).

\section{Discussion}

We hypothesized that FHR recovery would be shorter (quicker) in fetuses exposed to maternal exercise relative to those not exposed to maternal exercise. We found significantly quicker FHR recovery times in the exercise group relative to the control group at both intensities. Similarly, we found significantly more exercise exposed fetuses recovered by 20 min compared to fetuses of controls. Interestingly, control fetuses had significant increases in FHR response to exercise, whereas fetuses of exercisers did not. Participants in the EG had sig- 
nificantly quicker maternal HR recovery time after the three-minute walking test at $40 \%$ intensity. Furthermore, there are trends towards significant differences in the maternal HR recovery time after the walking test at $60 \%$ intensity.

\section{FHR variables}

These findings are the first to demonstrate $100 \%$ of fetuses exposed to exercise at recommended levels have a quicker recovery to baseline after maternal exercise compared to fetuses not exposed to exercise in utero. The significant difference in fetal recovery time of approximately 5-6 min between groups is similar to previous study findings (Roldan et al., 2015), which shows reproducibility of this result.

Post-exercise recovery time between 5 to $7 \mathrm{~min}$ in CG foetuses is similar to previous research (Barakat et al., 2008). Similar to our EG, Rafla and Cook (1999) reported lower FHR immediately after maternal exercise in fetuses from active women relative to fetuses of sedentary women. However, Rafla and Cook did not measure recovery time of FHR.

A decreased HR recovery time after exercise is an indicator of ANS adaptation in adults exposed to chronic aerobic exercise training (Barak et al., 2011; Barbosa et al., 2015; Carter, Banister, \& Blaber, 2003; Danieli et al., 2014; Wilmore \& Costill, 2007). Therefore, a faster recovery time in fetuses from EG supports the idea of fetal ANS adaptation to chronic maternal exercise exposure. This finding is aligned with previous findings from May et al. (2010) which also found measures indicative of a more mature ANS function in fetuses from women who exercised during pregnancy. The finding that all EG fetuses recovered more quickly than CG fetuses after maternal exercise supports the positive influence of maternal exercise on fetal cardiac ANS development and thus the Barker hypothesis related to health outcomes and not just disease outcomes of offspring (Barker, 2007). This faster recovery could have occurred in EG fetuses due to a significantly lower FHR after maternal exercise in comparison with CG fetuses, as it can be seen after the $40 \%$ walking test.
Although, after $60 \%$ walking test the between group difference in FHR after maternal effort is not statistically significant, showing a similar fetal cardiovascular response to the $60 \%$ walking test. Moreover, as it has been discussed above, FHR recovery after this test was significantly faster in fetuses exposed to chronic exercise. This fact also supports the positive influence of maternal exercise on fetal cardiac response.

Resting baseline FHR was lower in the EG compared to CG group, although this was not significant, most likely due to not controlling for fetal activity state (May et al., 2010; Schneider et al., 2009). Fetal state can influence baseline FHR, either increasing baseline FHR in active states or decreasing baseline FHR in the quiet states (Schneider et al., 2009). Therefore, analysing baseline FHR without controlling fetal state could have confounded this result.

There were significant differences between resting to post-maternal exercise FHR in fetuses of controls and not in fetuses of exercisers (- Table 2). Lower HR after submaximal exercise is one of the main cardiovascular adaptations to aerobic exercise in young adults (Calderón, 2001; Carter et al., 2003; Cornelissen, Verheyden, Aubert, \& Fagard, 2010; Wilmore \& Costill, 2007). Although it is not completely understood, this adaptation is generally attributed to a reduction in the sympathetic activation during submaximal exercise (Carter et al., 2003). Therefore, a potential explanation for our finding is that it may demonstrate a fetal heart adaptation to chronic maternal exercise exposure similar to the one occurring in young adults. The fact that FHR in EG fetuses did not increase significantly from resting to post-exercise period could be due to the FHR responding closely to baseline levels and not being affected by a maternal effort as a consequence of the chronic exposure to exercise. Nevertheless, this FHR response could be also due to a flat or reduce response to the FHR. One research has been found with a similar exercise intervention to the one presented in our study (Brenner et al., 1999), which ease results comparison. Authors in this research found that FHR at rest and post maternal effort was not different in fetuses of women who exercise. Furthermore, this research shows an increase in FHR during maternal exercise, voiding the possibility of a flat response of FHR in these fetuses. To confirm an approximate response of FHR in our study to the FHR response found by Brenner et al. (1999) FHR during maternal exercise should have been evaluated. Nevertheless, we have been unable to provide that analysis due to recurrent signal loss in FHR recordings during both walking tests. Hence, the mechanisms through which fetuses in the EG had a lower FHR after maternal exercise still remains unclear.

A recent meta-analysis (Skow et al., 2018) studying FHR responses to maternal exercise concludes there is insufficient research to support a relevant response of FHR associated with chronic exercise. However, the studies included in the meta-analysis have different methods of exercise training (i.e. frequency, intensity, duration, type of exercise), which make a strong conclusion regarding FHR response to chronic maternal exercise difficult.

\section{Maternal variables}

The maternal HR changes in the current study are similar to the results reported by others, showing a slightly lower, but not significant, resting HR in the EG (Perales et al., 2016; May et al., 2016) with improved HR variability (May et al., 2016). However, it seems that HR adaptations due to aerobic exercise are more difficult to detect during pregnancy most likely due to the naturally increasing resting $\mathrm{HR}$, a sign of altered maternal ANS, as gestation progresses.

In relation to maternal HR recovery time, this result reached significance in the walking test at $40 \%$ showing a faster recovery in participants in the EG and, hence, an adaptation to aerobic exercise. This is the first study to demonstrate maternal HR recovery time in women who participated in an exercise program throughout gestation. However, studies evaluating cardiorespiratory physical fitness (CRF) reported higher CRF values in active pregnant women compared con- 
trols (Halse, Wallman, Dimmock, Newnham, \& Guelfi, 2015; Ramírez-Vélez et al., 2011). The current study also a found similar trend towards improved HR recovery in the EG compared to the CG. These findings also serve to confirm an exercise adaptation on maternal physiology.

\section{Limitations}

One study limitation was the recurrent FHR signal loss during maternal exercise, which made the FHR response during maternal exercise data unusable. Another limitation of the CTG is the difficulty evaluating FHR variability through the paper traces obtained from the recordings.

Despite of the limitations, novelty of this equipment comes from the possibility of testing FHR in a wireless environment. Using a wireless CTG equipment allows testing FHR responses in a realistic situation, as walking is, vs a laboratory situation. Hence, results obtained from this test could have been easily transferred to a situation more approximate to a daily activity. Although as it has been already mentioned, recurrent signal loss occurred during the walking section of the protocol.

Although we did not control for physical activities outside of the study protocol, the positive exercise response of women in the intervention group support their compliance with the study protocol. Though our study had positive findings, all participants were in the healthy range for body mass index (BMI); this makes the results difficult to generalize to the average pregnant population. Further research is needed to determine if similar results are seen in overweight and obese pregnant women.

\section{Summary}

These results support that supervised moderate intensity exercise during pregnancy at levels recommended by ACOG are associated with quicker FHR recovery time, which can be related to an improved fetal ANS function. Further research needs to be done to determine

if these findings are also observed after birth.

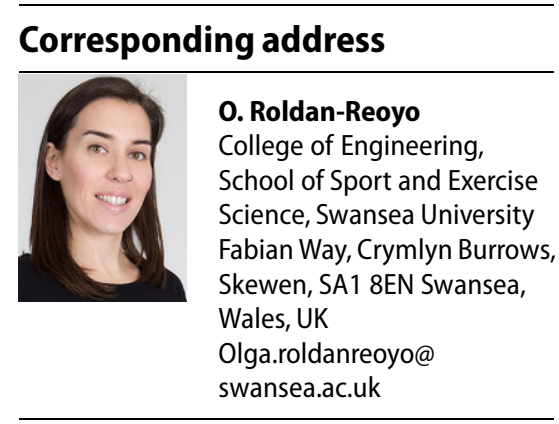

Acknowledgements. The authors acknowledge to committee of the three local hospitals for their help and support during the study.

\section{Compliance with ethical guidelines}

Conflict of interest O. Roldan-Reoyo, M. Pelaez, L. May and R. Barakat declare that they have no conflict of interests.

All procedures performed in studies involving human participants or on human tissue were in accordance with the ethical standards of the institutional and/or national research committee and with the 2013 Helsinki declaration and its later amendments or comparable ethical standards. Informed consent was obtained from all individual participants included in the study.

Open Access. This article is distributed under the terms of the Creative Commons Attribution 4.0 International License (http://creativecommons.org/licenses/by/ 4.0/), which permits unrestricted use, distribution, and reproduction in any medium, provided you give appropriate credit to the original author(s) and the source, provide a link to the Creative Commons license, and indicate if changes were made.

\section{References}

ACOG (2002). ACOG committee opinion. Exercise during pregnancy and the postpartum period. Number 267, January 2002. American College of Obstetricians and Gynecologists. Int.J Gynaecol Obstet, 77(1), 79-81.

ACOG (2015). Committee opinion. Physical activity and exercise during pregnancy and the postpartum period. Obstetrics \& Gynecology, 126(6), e135-e142.

ACSM (2014). ACSM's guidelines for exercise testing and prescription (9th edn.). Baltimore: Wolters Kluwer, Lippincott Williams \& Wilkins.

Armitage, P., \& Berry, G. (2002). Estadística para la investigación biomédica. Madrid: Harcourt Brace de España D.L.

Ayres-de-Campos, D., \& Bernardes, J. (2010). Twentyfive years after the FIGO guidelines for the use of fetal monitoring: time for a simplified approach? International Journal of Gynecology and Obstetrics, 110(1), 1-6. https://doi.org/10. 1016/j.jggo.2010.03.011.
Bahls, M., Sheldon, R. D., Taheripour, P., Clifford, K. A., Foust, K.B., Breslin, E.D., \& Newcomer, S.C. (2014). Mother's exercise during pregnancy programmes vasomotor function in adult offspring. Experimental Physiology, 99(1), 205-219. https://doi.org/10.1113/expphysiol. 2013.075978.

Barak, O., Ovcin, Z., Jakovljevic, D., Lozanov-Crvenkovic, Z., Brodie, D., \& Grujic, N. (2011). Heart rate recovery after submaximal exercise in four different recovery protocols in male athletes and non-athletes. Journal of Sports Science \& Medicine, 10(2), 369-375.

Barakat, R., Cordero, Y., Coteron, J., Luaces, M., \& Montejo, R. (2012a). Exercise during pregnancy improves maternal glucose screen at 24-28 weeks: a randomised controlled trial. British Journal of Sports Medicine, 46(9), 656-661. https://doi.org/10.1136/bjsports-2011-090009.

Barakat, R., Pelaez, M., Lopez, C., Montejo, R., \& Coteron, J. (2012b). Exercise during pregnancy reduces the rate of cesarean and instrumental deliveries: results of a randomized controlled trial. J Matern Fetal Neonatal Med, 25(11), 2372-2376.

Barakat, R., Ruiz, J.R., Rodríguez-Romo, G., MontejoRodríguez, R., \& Lucia, A. (2010). Does exercise training during pregnancy influence fetal cardiovascular responses to an exercise stimulus? Insights from a randomised, controlled trial. British Journal of Sports Medicine, 44(10), 762-764. https://doi.org/10.1136/bjsm.2009. 062547.

Barakat, R., Stirling, J., Zakynthinaki, M., \& Lucía, A. (2008). Acute maternal exercise during the third trimester of pregnancy, influence on foetal heart rate. Ejercicio físico durante el tercer trimestre de embarazo, influencia en la frecuencia cardiaca fetal. Introduction physical exercise becoming an integral. $R I C Y D E, I V(13), 33-43$.

Barbosa, T., Valente, L., Sobral, D., \& Barbosa, O. (2015). Relation between heart rate recovery after exercise testing and body mass index. Revista Poruguesa de Cardiologia, 34(1), 27-33.

Barker, D. J.P. (2007). The origins of the developmental origins theory. Journal of Internal Medicine, 261(5), 412-417. https://doi.org/10.1111/j. 1365-2796.2007.01809.x.

Bø, K., Artal, R., Barakat, R., Brown, W., Davies, G. A. L., Dooley, M., \& Khan, K. M. (2016). Exercise and pregnancy in recreational and elite athletes: 2016 evidence summary from the IOC expert group meeting, Lausanne. Part 1-exercise in women planning pregnancy and those who are pregnant. British Journal of Sports Medicine, 50(10), 571-589. https://doi.org/10.1136/ bjsports-2016-096218.

Brenner, I. K., Wolfe, L. A., Monga, M., \& McGrath, M.J. (1999). Physical conditioning effects on fetal heart rate responses to graded maternal exercise. Medicine and Science in Sports and Exercise, 31(6), 792-799.

Calderón, F.J. (2001). Fisiologia aplicada al deporte. Madrid:Tébar.

Carter, J.B., Banister, E.W., \& Blaber, A.P. (2003). Effect of endurance exercise on autonomic control of heart rate. Sports Medicine (Auckland, N.Z.), 33(1), 33-46. https://doi.org/10.2165/ 00007256-200333010-00003.

Cornelissen, V. A., Verheyden, B., Aubert, A. E., \& Fagard, R. H. (2010). Effects of aerobic training intensity on resting, exercise and post-exercise blood pressure, heart rate and heart-rate variability. Journal of Human Hypertension, 24(3), 175-182. https://doi.org/10.1038/jhh.2009.51. 
Danieli, A., Lusa, L., Potočnik, N., Meglič, B., Grad, A., \& Bajrović, F. (2014). Resting heart rate variability and heart rate recovery after submaximal exercise. Clinical Autonomic Research, 24(2), 53-61. https://doi.org/10.1007/s10286-0140225-2.

Evenson, K., Barakat, R., \& Brown, W. (2014). Guidelines for physical activity during pregnancy: comparisons from around the world. Am J Lifestyle Med, 8, 102-121.

Halse, R. E., Wallman, K. E., Dimmock, J. A., Newnham, J.P., \& Guelfi, K. J. (2015). Home-based exercise improves fitness and exercise attitude and intention in women with GDM. Medicine \& Science in Sports \& Exercise, 47(8), 1698-1704. https://doi. org/10.1249/MSS.0000000000000587.

Heffernan, K. S., Fahs, C. A., Shinsako, K. K., Jae, S. Y., \& Fernhall, B. (2007). Heart rate recovery and heart rate complexity following resistance exercise training and detraining in young men. American Journal of Physiology. Heart and Circulatory Physiology, 293, H3180-H3186. https://doi.org/10.1152/ajpheart.00648.2007.

May, L., Allen, J. J. B., \& Gustafson, K. (2016). Fetal and maternal cardiac responses to physical activity and exercise during pregnancy. Early Human Development, 94, 49-52. https://doi.org/10. 1016/j.earlhumdev.2016.01.008.

May, L. E., Glaros, A., Yeh, H.-W., Clapp, J. F., \& Gustafson, K. M. (2010). Aerobic exercise during pregnancy influences fetal cardiac autonomic control of heart rate and heart rate variability. Early Human Development, 86(4), 213-217. https://doi.org/ 10.1016/j.earlhumdev.2010.03.002.

May, L. E., Knowlton, J., Hanson, J., Suminski, R., Paynter, C., Fang, X., \& Gustafson, K. M. (2016). Effects of exercise during pregnancy on maternal heart rate and heart rate variability. $P M$ and $R, 8(7)$, 611-617. https://doi.org/10.1016/j.pmrj.2015. 11.006.

Mottola, M. F., Davenport, M. H., Ruchat, S. M., Davies, G. A., Poitras, V. J., Gray, C. E., \& Zehr, L. (2018). 2019 Canadian guideline for physical activity throughout pregnancy. British Journal of Sports Medicine, 52(21), 1339-1346. https://doi.org/10. 1136/bjsports-2018-100056.

Ostojic, S.M., Markovic, G., Calleja-Gonzalez, J., Jakovljevic, D. G., Vucetic, V., \& Stojanovic, M. D. (2010). Ultra short-term heart rate recovery after maximal exercise in continuous versus intermittent endurance athletes. European Journal of Applied Physiology, 108, 1055-1059. https://doi.org/10.1007/s00421-009-1313-1.

Ostojic, S. M., Stojanovic, M. D., \& Calleja-Gonzalez, J. (2011). Ultra short-term heart rate recovery after maximal exercise: relations to aerobic power in sportsmen. Chinese Journal of Physiology, 54(2), 105-110. https://doi.org/10.4077/CJP.2011. AMM018.

Pelaez, M. (2011). Efecto del ejercicio físico durante el embarazo sobre la ganancia excesiva de peso y sus consecuencias. Madrid: Universidad Politécnica de Madrid.

Pelaez, M., Gonzalez-Cerron, S., Montejo, R., Barakat, R., Haakstad, L., \& Bø, K. (2011). Exercise in pregnant women and birth weight: a randomized controlled trial. BMC Pregnancy \& Childbirth, 11(1), 66. https://doi.org/10.1186/1471-239311-66.

Perales, M., Santos-Lozano, A., Sanchis-Gomar, F. Luaces, M., Pareja-Galeano, H., Garatachea, N., \& Lucia, A. (2016). Maternal cardiac adaptations to a physical exercise program during pregnancy.
Med Sci Sports Exerc, 48(5), 896-906. https://doi. org/10.1249/MSS.0000000000000837.

Rafla, N. M., \& Cook, J. R. (1999). The effect of maternal exercise on fetal heart rate. Journal of Obstetrics and Gynaecology: The Journal of the Institute of Obstetrics and Gynaecology, 19(4), 381-384. https://doi.org/10.1080/01443619964698.

Ramírez-Vélez, R., Aguilar de Plata, A.C., Escudero, M. M., Echeverry, I., Ortega, J.G., Salazar, B., \& López-Jaramillo, P. (2011). Influence of regular aerobic exercise on endothelium-dependent vasodilation and cardiorespiratory fitness in pregnant women. Journal of Obstetrics and Gynaecology Research, 37(11), 1601. https://doi. org/10.1111/j.1447-0756.2011.01582.x.

RCOG (2001). The use of electronic fetal monitoring: The use and interpretation of cardiotocography in intrapartum fetal surveillance. Evidence Based Clinical Guideline, number 8 . London: RCOG Press. https://doi.org/10.1017/ CBO9781107415324.004

Roldan, O., Perales, M., Mateos, S., \& Barakat, R. (2015). Supervised physical activity during pregnancy improves fetal cardiac response. Rev. Int. Med. Cienc. Act. Fis. Deporte, 15(60), 757-772.

Sadler, T.W. (2010). Langman. Embriología médica. Barcelona: Wolters KI.

Schneider, U., Schleussner, E., Fiedler, A., Jaekel, S., Liehr, M., Haueisen, J., \& Hoyer, D. (2009). Fetal heart rate variability reveals differential dynamics in the intrauterine development of the sympathetic and parasympathetic branches of the autonomic nervous system. Physiological Measurement, 30(2), 215-226. https://doi.org/ 10.1088/0967-3334/30/2/008.

Skow, R. J., Davenport, M.H., Mottola, M. F., Davies, G. A., Poitras, V. J., Gray, C.E., \& Ruchat, S.-M. (2018). Effects of prenatal exercise on fetal heart rate, umbilical and uterine blood flow: a systematic review and meta-analysis. $\mathrm{Br} J$ Sports Med, 0, 1-12. https://doi.org/10.1136/ bjsports-2018-099822.

Szymanski, L. M., \& Satin, A. J. (2012). Exercise during pregnancy: fetal responses to current public health guidelines. Obstetrics and Gynecology, 119(3), 603-610.

Wilmore, J., \& Costill, D. (2007). Fisiología del esfuerzo y del deporte. $\left(6^{\circ}\right)$. Badalona: Paidotribo

Zavorsky, G.S., \& Longo, L.D. (2011). Exercise guidelines in pregnancy: new perspectives. Sports Medicine, 41(5), 345-360. https://doi.org/ 10.2165/11583930-000000000-00000. 